\title{
Existe alguma diferença entre um marcador esférico e uma moeda simples para planejamento digital de substituição de quadril?*
}

\section{Is There Any Difference Between a Spherical Marker and a Simple Coin for Hip Replacement Digital Planning?*}

\author{
Giancarlo Cavalli Polesello ${ }^{10}$ Thiago Tronco Salerno ${ }^{10}$ Marcelo Cavalheiro de Queiroz ${ }^{10}$ \\ Walter Ricioli Junior ${ }^{10}$ Nayra Deise Anjos Rabelo ${ }^{10}$ Emerson Kyioshi Honda ${ }^{10}$
}

\footnotetext{
${ }^{1}$ Departamento de Ortopedia e Traumatologia da Irmandade da Santa Casa de Misericórdia de São Paulo (ISCMSP), São Paulo, SP, Brasil
}

Rev Bras Ortop 2022;57(4):560-568.

\begin{abstract}
Endereço para correspondência Giancarlo Cavalli Polesello, M.D, Ph. D., Professor Adjunto e Chefe do grupo de quadril, Departamento de Ortopedia e Traumatologia da Santa Casa de São Paulo, Pavilhão Fernandinho Simonsen, Sala do Grupo do Quadril ( $2^{\circ}$ andar), Rua Dr. Cesário Motta Junior, 112, Bairro Vila Buarque, São Paulo, SP, Brasil (e-mail: giancarlopolesello@hotmail.com).
\end{abstract}

\author{
Resumo \\ Palavras-chave \\ - artroplastia de quadril \\ - prótese de quadril \\ - radiografia \\ - pelve \\ - ampliação \\ radiográfica
}

Objetivo Avaliar a precisão e as diferenças entre 2 tipos de marcadores metálicos, esfera e moeda, para calibração radiográfica no planejamento pré-operatório da artroplastia de quadril.

Métodos Quatro marcadores metálicos esféricos e quatro moedas, ambas de $25 \mathrm{~mm}$ de diâmetro, foram colocadas em trocânter maior, sínfise púbica, entre as coxas e a mesa do exame, para exame radiográfico do quadril em 33 pacientes com prótese de quadril. A cabeça da prótese foi utilizada para calibração e dois examinadores mediram os diâmetros da imagem dos marcadores, e os resultados foram analisados estatisticamente.

Resultados No trocânter maior, a esfera e a moeda não foram visualizadas em 19 radiografias $(57,6 \%)$. Entre as coxas, o marcador de moeda não foi visualizado em 13 radiografias (39,4\%). No trocânter maior, a precisão de $25 \mathrm{~mm}$ da moeda e da esfera foi, respectivamente, entre 57,1 e $63,3 \%$ e entre 64,3 e $92,9 \%$. A moeda entre as coxas atingiu $25 \mathrm{~mm}$ de precisão entre 50 e $60 \%$. Sobre a mesa de exame, os marcadores de moeda e esfera atingiram, respectivamente, diâmetros médios de $22,91 \mathrm{~mm}$ e $23 \mathrm{~mm}$, o menor coeficiente de variação, o menor intervalo de confiança e o posicionamento mais fácil. Houve diferença estatística entre as avaliações dos marcadores (moeda vs.

* Trabalho realizado no Grupo de Quadril do Departamento de Ortopedia e Traumatologia da Faculdade de Ciências Médicas Santa Casa de Misericórdia de São Paulo (FCMSCSP), São Paulo, SP, Brasil.

recebido

03 de Junho de 2020

aceito

14 de Outubro de 2020

Publicado on-line

de Janeiro 24, 2022
DOI https://doi.org/ $10.1055 / \mathrm{s}-0041-1724079$ ISSN 0102-3616.
(C) 2022. Sociedade Brasileira de Ortopedia e Traumatologia. All rights reserved.

This is an open access article published by Thieme under the terms of the Creative Commons Attribution-NonDerivative-NonCommercial-License, permitting copying and reproduction so long as the original work is given appropriate credit. Contents may not be used for commercial purposes, or adapted, remixed, transformed or built upon. (https://creativecommons.org/ licenses/by-nc-nd/4.0/)

Thieme Revinter Publicações Ltda., Rua do Matoso 170, Rio de Janeiro, RJ, CEP 20270-135, Brazil 


\begin{abstract}

\section{Keywords}

- arthroplasty, replacement, hip

- hip prosthesis

- radiography

- pelvis

- radiographic magnification

Objective To evaluate the accuracy and differences between 2 types of metallic markers, sphere, and coin, for radiographic calibration in the preoperative planning of hip arthroplasty.

Methods Four spherical metallic markers and four coins, both $25 \mathrm{~mm}$ in diameter, were placed on the greater trochanter, pubic symphysis, between the thighs, and on the table of the exam, for radiographic examination of the hip in 33 patients with hip prosthesis. The prosthesis head was used for calibration and two examiners measured the markers' image diameters, and the results were analyzed statistically.

Results In the greater trochanter, the sphere and the coin were not visualized in 19 radiographs (57.6\%). Between the thighs, the coin marker was not visualized in 13 radiographs (39.4\%). In the greater trochanter, the $25-\mathrm{mm}$ accuracy of the coin and the sphere was, respectively, between 57.1 and $63.3 \%$ and between 64.3 and $92.9 \%$. The coin between the thighs reached $25-\mathrm{mm}$ accuracy in between 50 and $60 \%$ of cases. Over the exam table, the coin and sphere markers reached, respectively, the mean diameters of $22.91 \mathrm{~mm}$ and $23 \mathrm{~mm}$, the lowest coefficient of variation, the lowest confidence interval, and the easiest positioning. There was statistical difference between the evaluations of the markers (coin vs. sphere) in all positions $(p<0.032)$, except for the exam table position $(p=0.083)$.

Conclusions The coin between the thighs is the best marker for radiographic calibration in the preoperative planning of hip arthroplasty, and we suggest the use of another coin on the exam table for comparison, considering the $8 \%$ reduction in relation to its real size.
\end{abstract}

esfera) em todas as posições ( $p<0,032)$, com exceção da posição na mesa de exame $(p=0,083)$.

Conclusões A moeda entre as coxas é o melhor marcador para calibração radiográfica no planejamento pré-operatório da artroplastia de quadril, e sugerimos o uso de outra moeda na mesa de exame para comparação, considerando os $8 \%$ de redução em relação ao seu tamanho real.

\section{Introdução}

O planejamento cirúrgico pré-operatório é fundamental para prever dificuldades durante o procedimento, tamanhos de componentes e posicionamento de implantes na artroplastia do quadril. ${ }^{1-6} 0$ planejamento cirúrgico adequado reduz o número de complicações associadas à discrepância entre os membros, mau posicionamento, afrouxamento precoce do implante, instabilidade, fratura periprotéstica e perda de massa óssea. ${ }^{1-6}$ Isso era tradicionalmente realizado com radiografias impressas e uso de transparências com ampliação estabelecida pelo fabricante da prótese, com variações habituais na faixa de 100 a $130 \%$ do tamanho real. ${ }^{5}$ No entanto, com o desenvolvimento da imagem radiográfica digital, o planejamento informatizado tornou-se uma alternativa prática com reprodutibilidade comprovada. ${ }^{7}$

Para corrigir a ampliação da radiografia digital, marcadores radiopacos, como esferas, ${ }^{8-10}$ discos (moedas) ${ }^{11}$ e placas $^{12,13}$ vêm sendo usados nos últimos 10 anos. ${ }^{9,10,14-17}$ Quanto mais perto da posição em relação à articulação estudada, menor será a variação na ampliação do marcador, ${ }^{17}$ diminuindo os erros de calibração. Para a artroplastia do quadril, os marcadores são comumente posicionados na região ao redor da articulação, como no trocânter maior $^{9,11,12,14,15,17}$ e entre as coxas. ${ }^{9,10,12-14,16,17}$

A calibração é um primeiro passo essencial do planejamento digital, realizado pelo examinador, identificando uma distância conhecida entre dois pontos ou pelo diâmetro de um círculo circunscrito em torno de três pontos periféricos do objeto de referência. A calibração padrãoouro é calculada em pacientes em acompanhamento pósoperatório a partir da cabeça da prótese implantada. Ainda não há padronização nem da melhor posição ou melhor tipo de marcador para o planejamento da artroplastia do quadril.

O objetivo deste artigo é avaliar a precisão e as diferenças entre dois tipos de marcadores metálicos, esfera e moeda, para calibração radiográfica no planejamento préoperatório da artroplastia do quadril. Nossa hipótese era de que a esfera posicionada ao lado do trocânter seria o melhor método de calibração devido à proximidade anatômica à articulação do quadril e à instalação de calibração, mas o marcador da moeda teria desempenho semelhante ao manter seu maior diâmetro sempre visível na radiografia. 


\section{Materiais e métodos}

Este estudo transversal prospectivo foi realizado pelo Grupo de Cirurgia de Quadril do Departamento de Ortopedia e Traumatologia da nossa instituição.

O estudo foi realizado com uma amostra de conveniência de 33 pacientes de ambos os sexos, com prótese total e/ou parcial do quadril, primária ou revisão, que foram submetidos à radiografia pélvica como parte do acompanhamento pós-operatório em abril e maio de 2017. Radiografias em que o diâmetro da cabeça da prótese era desconhecido nos registros hospitalares ou que estavam fora dos padrões radiográficos foram excluídas do estudo. ${ }^{18}$

\section{Procedimentos}

Todos os pacientes foram submetidos à radiografia pélvica na incidência anteroposterior (AP), realizada com o paciente em decúbito dorsal, com rotação interna dos quadris entre 15 e $20^{\circ}$ e raio incidente na linha média, logo acima da sínfise púbica. ${ }^{18}$ Para a padronização, a distância do filme focal foi de $100 \mathrm{~cm}$, determinada com uma fita métrica e indicador de luz Optimus 50 (Philips Medical Systems, Hamburgo, Alemanha). Para padronizar a análise das radiografias, o cóccix foi alinhado com a sínfise púbica (com distância entre elas de $2,5 \mathrm{~cm}$ para mulheres e $1,5 \mathrm{~cm}$ para homens), bem como simetria dos foramens obturadores. ${ }^{18}$

Antes da radiografia, um único médico posicionou quatro esferas ou quatro moedas em quatro posições ao redor da pelve. $O$ diâmetro de ambos os marcadores foi de $25 \mathrm{~mm}$, confirmado por um paquimetro analógico Mitutoyo p-06 (Mitutoyo Sul Americana, Suzano, SP, Brasil) e uma pinça composta de fibra de carbono WesternPRO (Pasadena, CA, EUA), ambas certificadas pelo Instituto Nacional de Metrologia do Brasil (INMETRO). Os marcadores foram mantidos no lugar com fita adesiva ou um tubo de cloreto de polivinil claro (PVC) com comprimento de $3 / 4$ polegadas, como descrito em um estudo anterior, ${ }^{19}$ e um conduíte elétrico flexível de PVC
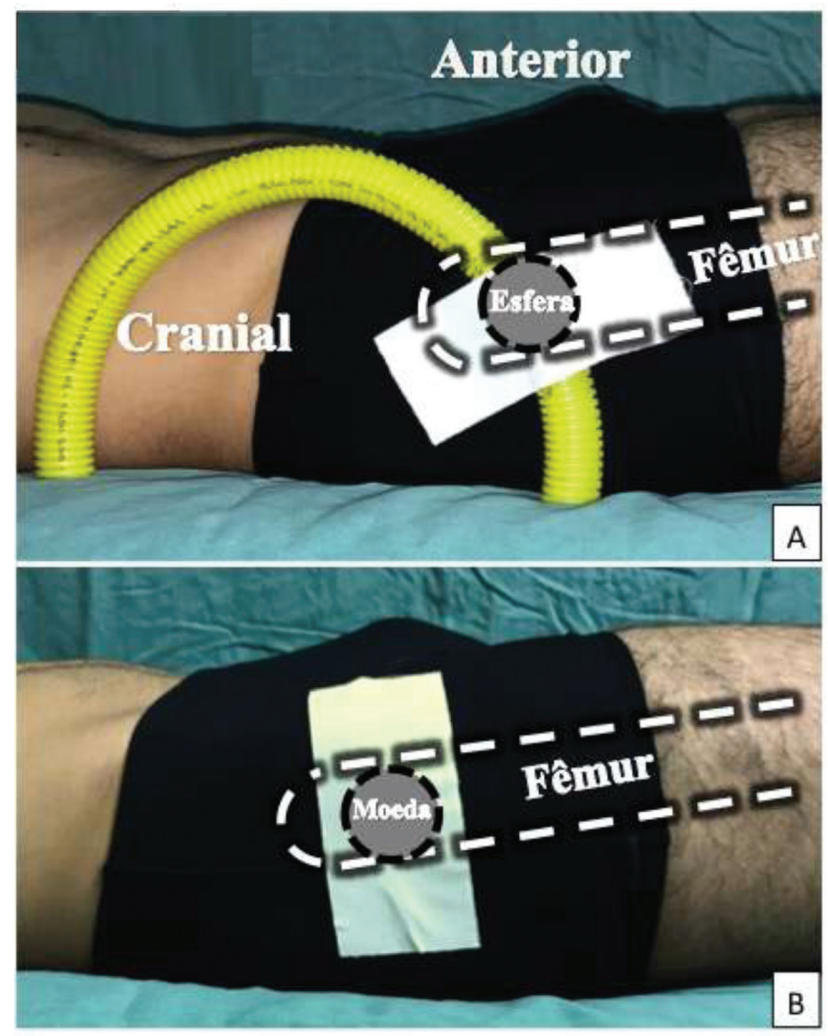

Fig. 1 Posicionamento da esfera no trocânter mais à direita (1A); Posicionamento da moeda no trocânter mais à direita (1B).

do mesmo comprimento. A mangueira e o conduíte foram cortados longitudinalmente para permitir que o deslizamento controlado da esfera ajustasse a posição. Os marcadores foram posicionados nos seguintes locais: 1-Além do trocânter, na pele, com a esfera no conduíte elétrico e a moeda anexada com fita no mesmo nível(-Figs. 1A e 1B); 2 Entre as coxas, no plano do trocânter maior, com a esfera na mangueira colocada o mais próxima possível e a moeda no mesmo nível(-Figs. 2A e 2B); 3-Ao nível da borda
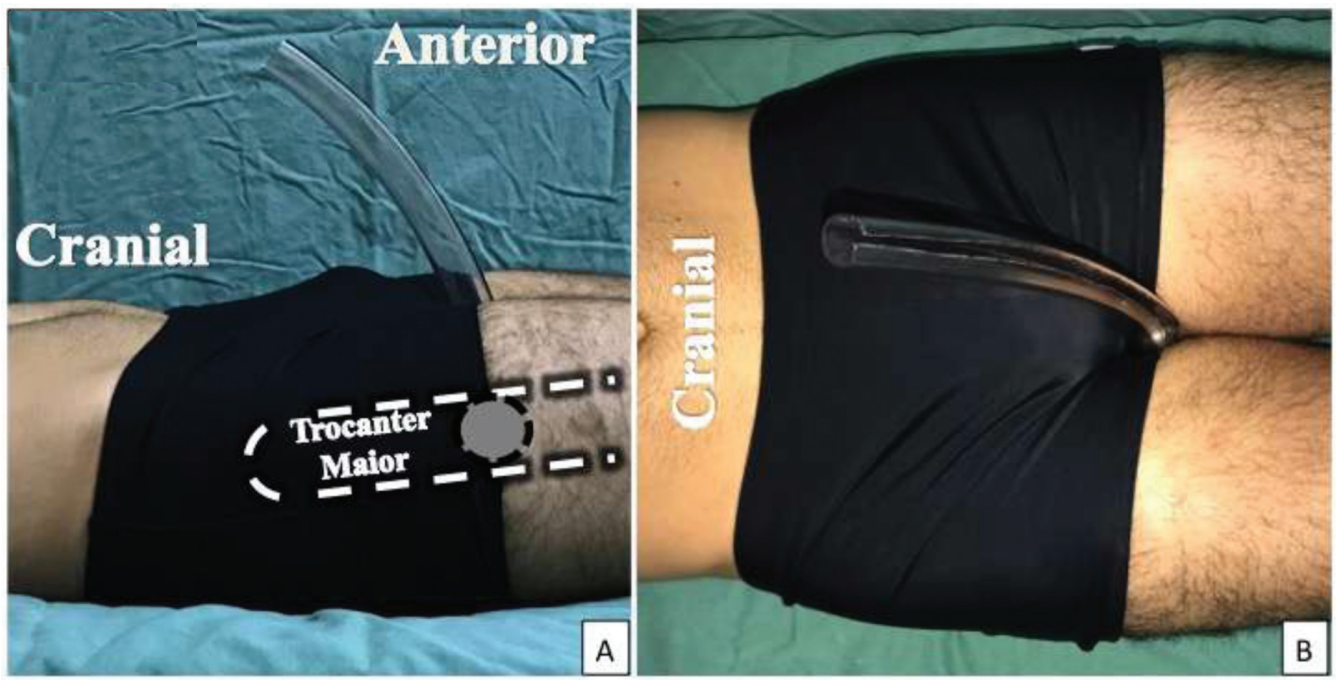

Fig. 2 Posicionamento da esfera entre as coxas, no plano do trocânter maior, vista lateral (2A) e vista superior (2B). 


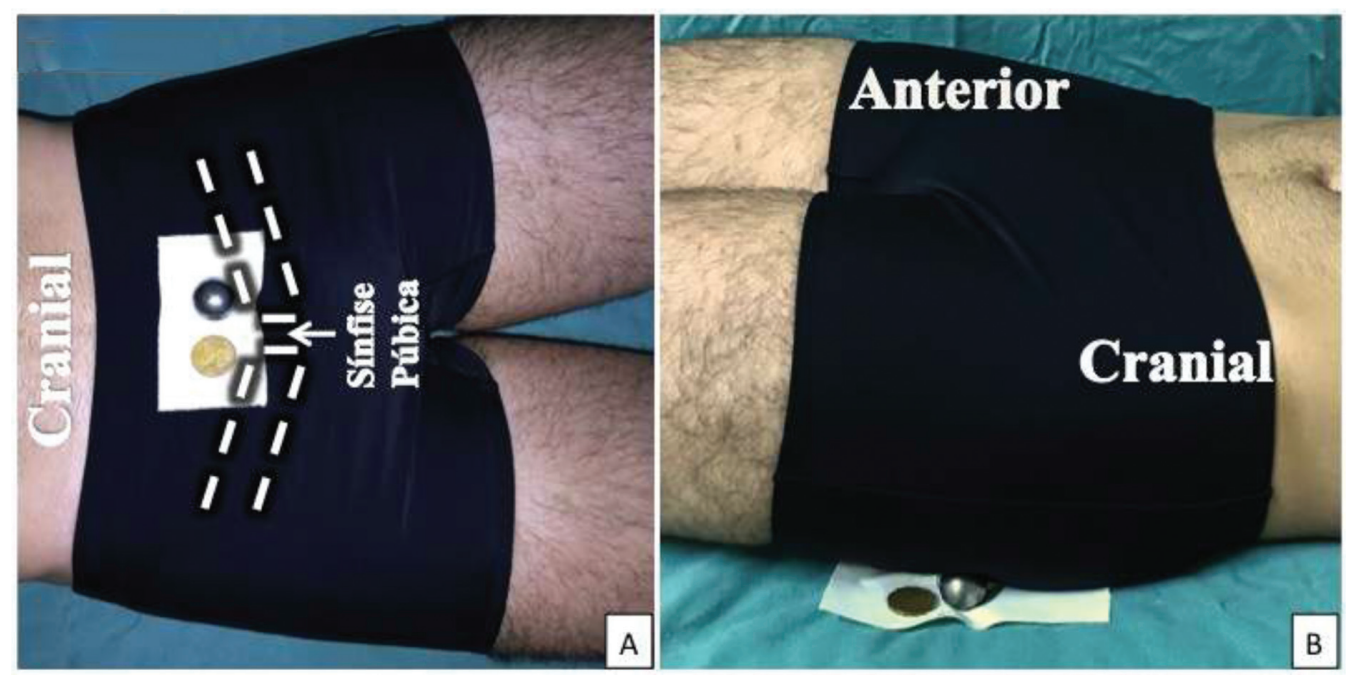

Fig. 3 Posicionamento da esfera e da moeda na sínfise púbica (3A); Posicionamento da esfera e da moeda na mesa de exame, no lado esquerdo do paciente (3B).

anterosuperior da sínfise púbica, ambas anexadas com fita adesiva (-Fig. 3A); 4-Entre a mesa de exame e o segmento proximal da coxa esquerda, $4 \mathrm{~cm}$ distal ao trocânter maior, ambos ligados com fita adesiva (-Fig. 3B).

\section{Análise das imagens}

Consideramos apenas radiografias que permitiram a medição digital da cabeça do componente protético e pelo menos dois marcadores e moedas esféricas (-Figs. 4A, 4B, 4C e 4D) na mesma imagem. Não foram analisados marcadores não visualizados na radiografia.

As imagens digitais foram analisadas por dois médicos que receberam treinamento prévio para uso do software de planejamento AGFA HealthCare - IMPAX Ortopaedic Tools (AGFA Healthcare, Greenville, SC, EUA). A cabeça da prótese foi usada como parâmetro de calibração com base em três pontos marginais e confirmada com um círculo, ${ }^{8-10,17}$ uma vez que o diâmetro é conhecido e o local está centrado na articulação.

Os diâmetros dos marcadores após a calibração radiográfica foram medidos pela mesma técnica de identificação de três pontos periféricos e formação de um círculo para confirmar o diâmetro do marcador, tanto para a esfera ${ }^{9}$ quanto para a moeda. ${ }^{11} \mathrm{O}$ diâmetro da esfera foi avaliado considerando todo o círculo preenchido, enquanto para a moeda foi considerado o maior diâmetro da imagem.

Cada avaliador mediu todas as imagens das moedas e esferas visualizadas em cada posição para posterior comparação entre as medidas. Todas as análises foram realizadas com um único computador HP Pavilion DV7 (The HewlettPackard Company, Palo Alto, CA, EUA).

\section{Análise estatística}

As características antropométricas da amostra foram descritas pelo cálculo dos meios e desvios-padrão, bem como as frequências absolutas e relativas.
O teste $\mathrm{t}$ de uma amostra foi utilizado para comparar a média em cada posição para cada avaliação dos examinadores com o valor de referência de $25 \mathrm{~mm}$. Utilizou-se o teste $\mathrm{t}$ de Student emparelhado, e a média, desvio-padrão, e coeficiente de variação - mínimo e máximo - de cada avaliação dos examinadores foram calculados em cada posição para julgar a semelhança entre os marcadores.

As estatísticas descritivas em percentual do valor real (25 mm) foram avaliadas pela média, mediana, desviopadrão, intervalo mínimo, máximo e de confiança. Também foi calculada a precisão de cada marcador em cada posição. A igualdade de duas proporções foi aplicada para caracterizar a distribuição relativa da frequência das variáveis qualitativas. Os resultados foram comparados pela análise de variância (ANOVA) e pelos testes de Pearson e do qui-quadrado.

Todas as análises foram realizadas com os programas IBMSPSS para Windows versão 20.0 (IBM Corp., Armonk, NY, EUA), Minitab 16 (Minitab LLC, State College, PA, EUA) e Microsoft Excel 2010 (Microsoft Corp., Redmond, WA, EUA), com um nível de significância de $5 \%$, seguindo a literatura de referência. ${ }^{20-22}$

\section{Resultados}

Os valores médios de ambos os marcadores no trocânter maior e entre as coxas estavam próximos do valor real de $25 \mathrm{~mm}$. Não houve diferença dos valores médios na posição de trocanter maior com o valor real, mas com a posição entre as coxas, apenas a moeda obteve uma diferença estatística e foi considerada mais precisa. A - Tabela 1 relata as avaliações dos dois examinadores em comparação com o tamanho real de $25 \mathrm{~mm}$.

A comparação entre os achados dos examinadores para cada marcador nas diferentes posições, como mostra a - Tabela 2, revela semelhança das seguintes medidas: moeda no trocânter maior, entre coxas e posições de sínfise púbica; e esfera nas posições de sínfise púbica e mesa de exame.

A - Tabela 3 demonstra uma diferença estatística das medidas entre os marcadores (moeda vs. esfera) em todas as posições, exceto na mesa de exame (valor de $p=0,083$ ). 

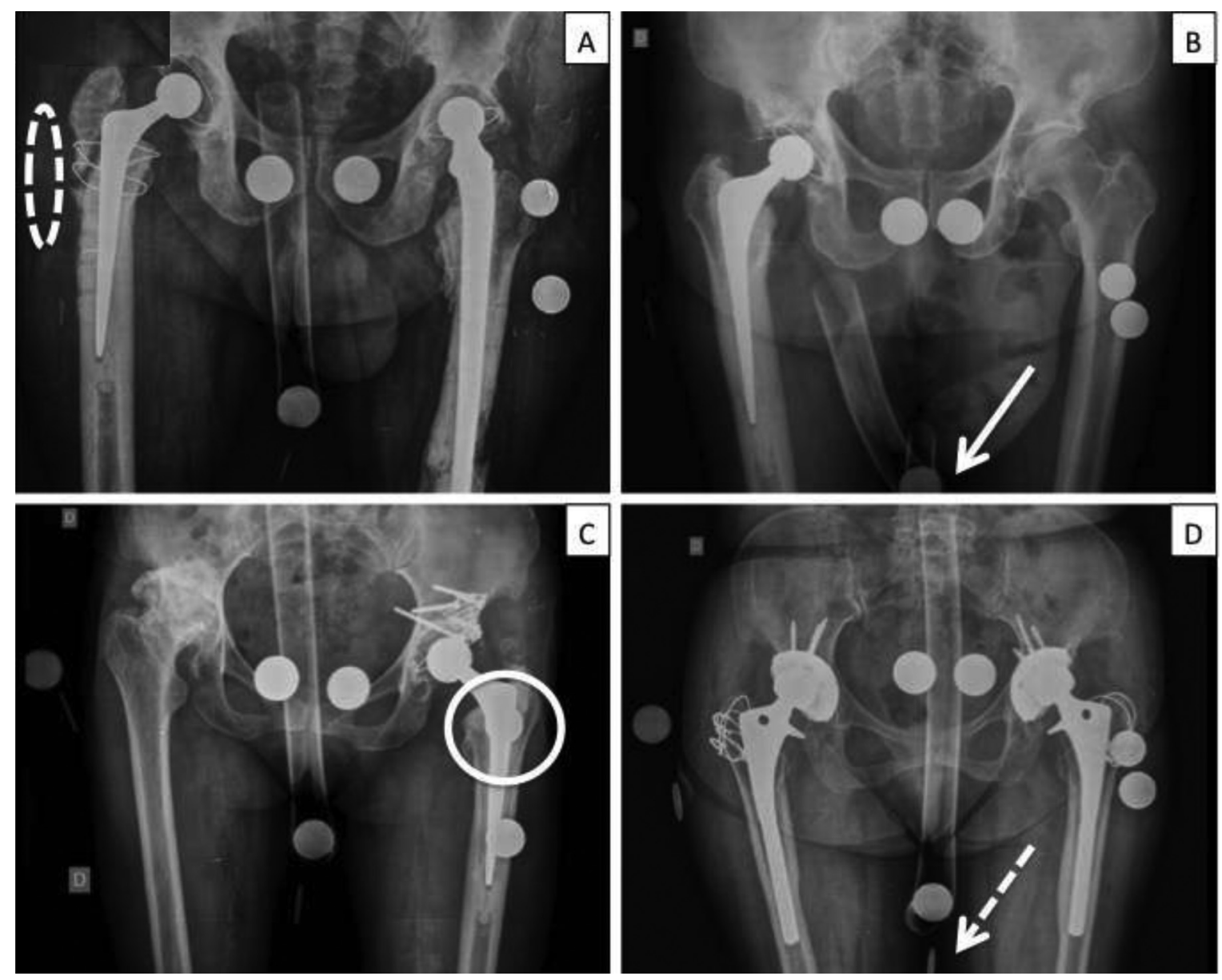

Fig. 4 Quatro radiografias da amostra analisada. - Figura 4A, note a ausência de marcadores no trocânter maior (círculo de linha tracejada). Nos pacientes do sexo masculino (- Figs. 4A e 4B), os marcadores entre as coxas estão no limite inferior da imagem, mas o abdome em avental devido à grande hérnia abdominal na - Figura 4B impede o posicionamento correto dos marcadores, e a moeda é difícil de visualizar entre as coxas (seta branca). A sobreposição da moeda com o eixo femoral na - Figura $\mathbf{4 C}$ (círculo) não impede a calibração, mas dificulta a técnica. A visualização parcial da moeda quando colocada verticalmente no trocânter maior ou entre as coxas, como retratado na - Figura 4D (seta de linha tracejada), impede a medição do diâmetro maior, impossibilitando a calibração.

A - Tabela 4 identifica os valores percentuais em relação ao tamanho real de $25 \mathrm{~mm}$ para permitir compreender a faixa de variação medida em cada posição dos marcadores.

A precisão calculada para cada marcador nas várias posições por cada examinador é mostrada na - Tabela 5. Com a moeda na mesa de exame, utilizando o diâmetro de $23 \mathrm{~mm}$ como referência, o examinador 1 alcançou precisão de 90,9\%(30 das 33 radiografias)e o examinador 2 obteve precisão de $78,8 \%$ (27 das 33 radiografias).

Houve maior perda de imagens de ambos os marcadores na posição do trocânter maior, correspondendo a 19 perdas em 33 radiografias (57,6\%). Nessa posição, para a amostra feminina houve 11 perdas em 18 radiografias (61,1\%), ao passo que o número para o sexo masculino foi de 8 perdas em 15 radiografias (53,3\%). Para a moeda colocada entre as coxas, houve 13 perdas em 33 radiografias (39,4\%), sendo a decomposição por sexo 8 perdas em 15 radiografias (53,3\%) para homens e 5 perdas em 18 radiografias (27,8\%) para as mulheres.

\section{Discussão}

O presente trabalho trata-se de um estudo transversal com o objetivo de avaliar a correção, precisão e diferenças no uso de dois marcadores metálicos (esfera e moeda) para calibrar a radiografia da pelve. Considerando correção, precisão, coeficiente de variação, inspeção visual das imagens e facilidade de posicionamento, a moeda colocada entre as coxas foi a melhor marca, com média de $25 \mathrm{~mm}$, mas sofreu grande perda de imagens (39,4\%). A moeda na mesa de exame apresentou média de $23 \mathrm{~mm}$, menor que o tamanho real, mas obteve um baixo coeficiente de variação (1\%) e apareceu em todas as imagens. Portanto, sugerimos o uso de moedas nas duas posições.

O melhor tipo de marcador radiográfico deve estar amplamente disponível, prático de posicionar, confiavelmente visível nas imagens e fácil de medir e calibrar com precisão. As dimensões da moeda são padronizadas e ela está disponível para qualquer serviço de radiologia que não tenha outro tipo de marcador. A identificação de marcadores quando posicionados no trocânter maior e sínfise púbica é suscetível a erros humanos, ao contrário da posição na mesa de exame, que é fácil de alcançar e livre desse tipo de erro.

Nas posições de sínfise púbica e mesa de exame, todas as imagens foram facilmente visualizadas. Apesar de pacientes obesos necessitarem de cuidados especiais no posicionamento 
Diferença entre um marcador esférico e uma moeda para planejamento de substituição de quadril Polesello et al. 565

Tabela 1 Comparação da posição/marcador com o valor real $(25 \mathrm{~mm})$ pelos examinadores

\begin{tabular}{|c|c|c|c|c|c|c|c|c|c|}
\hline \multicolumn{2}{|c|}{ Examinador 1} & Média (mm) & $\mathrm{DP}(\mathrm{mm})$ & CV & $\operatorname{Min}(\mathrm{mm})$ & $\operatorname{Max}(\mathrm{mm})$ & $\mathrm{N}$ & IC & Valor deP \\
\hline \multirow[t]{4}{*}{ Moeda } & Trocânter maior & 24,71 & 0,61 & $2 \%$ & 24 & 26 & 14 & 0,32 & 0,104 \\
\hline & Entre as coxas & 25 & 0,65 & $3 \%$ & 24 & 26 & 20 & 0,28 & 1 \\
\hline & Sínfise púbica & 27,7 & 0,47 & $2 \%$ & 27 & 28 & 33 & 0,16 & $<0,001$ \\
\hline & Mesa de exames & 22,91 & 0,29 & $1 \%$ & 22 & 23 & 33 & 0,1 & $<0,001$ \\
\hline \multirow[t]{4}{*}{ Esfera } & Trocânter maior & 25,07 & 0,27 & $1 \%$ & 25 & 26 & 14 & 0,14 & 0,336 \\
\hline & Entre as coxas & 25,58 & 0,5 & $2 \%$ & 25 & 26 & 33 & 0,17 & $<0,001$ \\
\hline & Sínfise púbica & 27,88 & 0,48 & $2 \%$ & 27 & 29 & 33 & 0,17 & $<0,001$ \\
\hline & Mesa de exames & 23 & 0,25 & $1 \%$ & 22 & 24 & 33 & 0,09 & $<0,001$ \\
\hline \multicolumn{2}{|c|}{ Examinador 2} & Média (mm) & $\mathrm{DP}(\mathrm{mm})$ & $\mathrm{CV}$ & $\operatorname{Min}(\mathrm{mm})$ & $\operatorname{Max}(\mathrm{mm})$ & $\mathrm{N}$ & IC & Valor deP \\
\hline \multirow[t]{4}{*}{ Moeda } & Trocânter maior & 24,79 & 0,58 & $2 \%$ & 24 & 26 & 14 & 0,3 & 0,189 \\
\hline & Entre as coxas & 25 & 0,73 & $3 \%$ & 24 & 26 & 20 & 0,32 & 1 \\
\hline & Sínfise púbica & 27,7 & 0,59 & $2 \%$ & 26 & 29 & 33 & 0,2 & $<0,001$ \\
\hline & Mesa de exames & 23,15 & 0,44 & $2 \%$ & 22 & 24 & 33 & 0,15 & $<0,001$ \\
\hline \multirow[t]{4}{*}{ Esfera } & Trocânter maior & 24,79 & 0,58 & $2 \%$ & 24 & 26 & 14 & 0,3 & 0,189 \\
\hline & Entre as coxas & 25,27 & 0,57 & $2 \%$ & 24 & 26 & 33 & 0,2 & 0,01 \\
\hline & Sínfise púbica & 27,97 & 0,53 & $2 \%$ & 27 & 29 & 33 & 0,18 & $<0,001$ \\
\hline & Mesa de exames & 22,97 & 0,3 & $1 \%$ & 22 & 24 & 33 & 0,1 & $<0,001$ \\
\hline
\end{tabular}

Abreviaturas: CV, coeficiente de variação; IC, intervalo de confiança; Kg, quilograma; Max, máximo; Min, mínimo; mm, milímetro; N, tamanho da amostra; Valor de $P$, significância.

Tabela 2 Comparação entre examinadores na mesma posição de cada marcador

\begin{tabular}{|c|c|c|c|c|c|c|c|c|c|}
\hline \multicolumn{2}{|l|}{ Moeda } & Média (mm) & $\mathrm{DP}(\mathrm{mm})$ & $\mathrm{CV}(\mathrm{mm})$ & $\operatorname{Min}(\mathrm{mm})$ & $\operatorname{Max}(\mathrm{mm})$ & $\mathrm{N}$ & IC & Valor $\mathrm{de} P$ \\
\hline \multirow[t]{2}{*}{ Trocânter maior } & Examinador 1 & 24,71 & 0,61 & $2 \%$ & 24 & 26 & 14 & 0,32 & 0,336 \\
\hline & Examinador 2 & 24,79 & 0,58 & $2 \%$ & 24 & 26 & 14 & 0,3 & \\
\hline \multirow[t]{2}{*}{ Entre as coxas } & Examinador 1 & 25 & 0,65 & $3 \%$ & 24 & 26 & 20 & 0,28 & 1 \\
\hline & Examinador 2 & 25 & 0,73 & $3 \%$ & 24 & 26 & 20 & 0,32 & \\
\hline \multirow[t]{2}{*}{ Sínfise púbica } & Examinador 1 & 27,7 & 0,47 & $2 \%$ & 27 & 28 & 33 & 0,16 & 1 \\
\hline & Examinador 2 & 27,7 & 0,59 & $2 \%$ & 26 & 29 & 33 & 0,2 & \\
\hline \multirow[t]{2}{*}{ Mesa de exames } & Examinador 1 & 22,91 & 0,29 & $1 \%$ & 22 & 23 & 33 & 0,1 & 0,003 \\
\hline & Examinador 2 & 23,15 & 0,44 & $2 \%$ & 22 & 24 & 33 & 0,15 & \\
\hline \multicolumn{2}{|l|}{ Esfera } & Média (mm) & $\mathrm{DP}(\mathrm{mm})$ & $\mathrm{CV}(\mathrm{mm})$ & $\operatorname{Min}(\mathrm{mm})$ & $\operatorname{Max}(\mathrm{mm})$ & $\mathrm{N}$ & IC & Valor de $P$ \\
\hline \multirow[t]{2}{*}{ Trocânter maior } & Examinador 1 & 25,07 & 0,27 & $1 \%$ & 25 & 26 & 14 & 0,14 & 0,04 \\
\hline & Examinador 2 & 24,79 & 0,58 & $2 \%$ & 24 & 26 & 14 & 0,3 & \\
\hline \multirow[t]{2}{*}{ Entre as coxas } & Examinador 1 & 25,58 & 0,5 & $2 \%$ & 25 & 26 & 33 & 0,17 & 0,006 \\
\hline & Examinador 2 & 25,27 & 0,57 & $2 \%$ & 24 & 26 & 33 & 0,2 & \\
\hline \multirow[t]{2}{*}{ Sínfise púbica } & Examinador 1 & 27,88 & 0,48 & $2 \%$ & 27 & 29 & 33 & 0,17 & 0,263 \\
\hline & Examinador 2 & 27,97 & 0,53 & $2 \%$ & 27 & 29 & 33 & 0,18 & \\
\hline \multirow[t]{2}{*}{ Mesa de exames } & Examinador 1 & 23 & 0,25 & $1 \%$ & 22 & 24 & 33 & 0,09 & 0,572 \\
\hline & Examinador 2 & 22,97 & 0,3 & $1 \%$ & 22 & 24 & 33 & 0,1 & \\
\hline
\end{tabular}

Abreviaturas: CV, coeficiente de variação; IC, intervalo de confiança; Max, máximo; Min, mínimo; mm, milímetro; N, tamanho da amostra; Valor de $P$, significância.

dos marcadores, que devem estar abaixo da dobra do abdômen na sínfise púbica e abaixo da coxa quando na mesa de exame, não ocorreram perdas de imagem com esses marcadores. As imagens da moeda entre as coxas em 13 radiografias (39,4\%) e de ambos os marcadores no trocânter maior em 19 radiografias (57,6\%) eram apenas parcialmente visíveis ou não visíveis, de modo que nenhum cálculo era possível. Houve diferença entre os sexos, com maior perda de imagens em pacientes do 
Tabela 3 Comparação dos marcadores para cada posição avaliada pelo examinador 1

\begin{tabular}{|c|c|c|c|c|c|c|c|c|c|}
\hline \multicolumn{2}{|l|}{ Examinador 1} & Média (mm) & $\mathrm{SD}(\mathrm{mm})$ & $\mathrm{CV}(\mathrm{mm})$ & $\operatorname{Min}(\mathrm{mm})$ & $\operatorname{Max}(\mathrm{mm})$ & $\mathrm{N}$ & IC & Valor de $P$ \\
\hline \multirow[t]{2}{*}{ Trocânter maior } & Moeda & 24,71 & 0,61 & $2 \%$ & 24 & 26 & 14 & 0,32 & \multirow[t]{2}{*}{0,019} \\
\hline & Esfera & 25,07 & 0,27 & $1 \%$ & 25 & 26 & 14 & 0,14 & \\
\hline \multirow[t]{2}{*}{ Entre as coxas } & Moeda & 25 & 0,65 & $3 \%$ & 24 & 26 & 20 & 0,28 & \multirow[t]{2}{*}{0,002} \\
\hline & Esfera & 25,55 & 0,51 & $2 \%$ & 25 & 26 & 20 & 0,22 & \\
\hline \multirow[t]{2}{*}{ Sínfise púbica } & Moeda & 27,7 & 0,47 & $2 \%$ & 27 & 28 & 33 & 0,16 & \multirow[t]{2}{*}{0,032} \\
\hline & Esfera & 27,88 & 0,48 & $2 \%$ & 27 & 29 & 33 & 0,17 & \\
\hline \multirow[t]{2}{*}{ Mesa de exames } & Moeda & 22,91 & 0,29 & $1 \%$ & 22 & 23 & 33 & 0,1 & \multirow[t]{2}{*}{0,083} \\
\hline & Esfera & 23 & 0,25 & $1 \%$ & 22 & 24 & 33 & 0,09 & \\
\hline
\end{tabular}

Abreviaturas: $\mathrm{CV}$, coeficiente de variação; DP, desvio padrão; IC, intervalo de confiança; Max, máximo; Min, mínimo; mm, milímetro; N, tamanho da amostra; Valor de $P$, significância.

Tabela 4 Diferenças percentuais em relação aos valores reais medidos pelo examinador 1

\begin{tabular}{|l|l|l|l|l|l|l|l|l|}
\hline \multicolumn{2}{|l|}{ Examinador 1} & Média & Mediana & DP & Min & Max & N & IC \\
\hline \multirow{4}{*}{ Moeda } & Trocânter maior & $1,14 \%$ & $0,00 \%$ & $2,44 \%$ & $-4,00 \%$ & $4,00 \%$ & 14 & $1,28 \%$ \\
\cline { 2 - 8 } & Entre as coxas & $0,00 \%$ & $0,00 \%$ & $2,60 \%$ & $-4,00 \%$ & $4,00 \%$ & 20 & $1,14 \%$ \\
\cline { 2 - 9 } & Sínfise púbica & $-10,79 \%$ & $-12,00 \%$ & $1,87 \%$ & $-12,00 \%$ & $-8,00 \%$ & 33 & $0,64 \%$ \\
\cline { 2 - 9 } & Mesa de exames & $8,36 \%$ & $8,00 \%$ & $1,17 \%$ & $8,00 \%$ & $12,00 \%$ & 33 & $0,40 \%$ \\
\hline \multirow{5}{*}{ Esfera } & Trocânter maior & $-0,29 \%$ & $0,00 \%$ & $1,07 \%$ & $-4,00 \%$ & $0,00 \%$ & 14 & $0,56 \%$ \\
\cline { 2 - 9 } & Entre as coxas & $-2,30 \%$ & $-4,00 \%$ & $2,01 \%$ & $-4,00 \%$ & $0,00 \%$ & 33 & $0,68 \%$ \\
\cline { 2 - 8 } & Sínfise púbica & $-11,52 \%$ & $-12,00 \%$ & $1,94 \%$ & $-16,00 \%$ & $-8,00 \%$ & 33 & $0,66 \%$ \\
\cline { 2 - 8 } & Mesa de exames & $8,00 \%$ & $8,00 \%$ & $1,00 \%$ & $4,00 \%$ & $12,00 \%$ & 33 & $0,34 \%$ \\
\hline
\end{tabular}

Abreviaturas: IC, intervalo de confiança; Max, máximo; Min, mínimo; N, tamanho da amostra; DP, desvio padrão.

Tabela 5 Precisão em relação a 25 mm dos marcadores para cada posição e examinador

\begin{tabular}{|c|c|c|c|c|c|c|}
\hline \multicolumn{2}{|c|}{ Precisão (25 mm) } & \multicolumn{2}{|c|}{ Examinador 1} & \multicolumn{2}{|c|}{ Examinador 2} & \multirow[t]{2}{*}{ Total } \\
\hline & & $\mathrm{N}$ & Precisão & $\mathrm{N}$ & Precisão & \\
\hline \multirow[t]{4}{*}{ Moeda } & Trocânter maior & 8 & $57,10 \%$ & 9 & $64,30 \%$ & 14 \\
\hline & Entre as coxas & 12 & $60,00 \%$ & 10 & $50,00 \%$ & 20 \\
\hline & Sínfise púbica & 0 & $0,00 \%$ & 0 & $0,00 \%$ & 33 \\
\hline & Mesa de exames & 0 & $0,00 \%$ & 0 & $0,00 \%$ & 33 \\
\hline \multirow[t]{4}{*}{ Esfera } & Trocânter maior & 13 & $92,90 \%$ & 9 & $64,30 \%$ & 14 \\
\hline & Entre as coxas & 14 & $42,40 \%$ & 20 & $60,60 \%$ & 33 \\
\hline & Sínfise púbica & 0 & $0,00 \%$ & 0 & $0,00 \%$ & 33 \\
\hline & Mesa de exames & 0 & $0,00 \%$ & 0 & $0,00 \%$ & 33 \\
\hline
\end{tabular}

Abreviação: N, tamanho da amostra.

sexo feminino no trocânter maior $(61,1 \%)$, provavelmente devido ao padrão de acúmulo de gordura posterolateral nos quadris, enquanto a perda foi maior para os pacientes do sexo masculino quando o marcador foi posicionado entre as coxas (53,3\%), provavelmente causada pelo volume genital nesta região. As imagens circulares da esfera e da moeda na mesa de exame e na sínfise púbica foram mais fáceis de medir. Considerando o coeficiente de variação inferior a 3\% para ambos os marcadores em todas as posições, podem ser considerados estáveis em relação à média.
Com relação à precisão, as medidas de moeda entre as coxas $(p=1.000)$, esfera no trocânter maior $(p=0,336)$ e moeda no trocânter maior $(p=0,189)$ foram as mais próximas do tamanho real de $25 \mathrm{~mm}$. No trocânter maior, as medidas de precisão da moeda e da esfera foram, respectivamente, entre 57,1 e $63,3 \%$ e entre 64,3 e $92,9 \%$. Entre as coxas, as medidas de precisão da moeda e da esfera foram entre 50 e $60 \%$ e 42,4 e $60 \%$, respectivamente. Mesmo não sendo preciso em $25 \mathrm{~mm}$, o resultado da moeda e da esfera na mesa de exame foi confiável, com o melhor intervalo de 
confiança (entre 0,09-0,1), denotando baixa variação da média, e a precisão para $23 \mathrm{~mm}$ foi entre 78,8 e $93,9 \%$.

Os marcadores, quando localizados ao nível da sínfise púbica e na mesa de exame, estiveram sujeitos a variação, devido à ampliação radiográfica do cone de emissão de objetos mais distantes do filme em comparação com objetos mais próximos. ${ }^{12,23}$ Para a calibração com esses marcadores, é necessário compreender essa influência para que erros não ocorram fora da margem entre $+3 \%$ e $-3 \%$, que é considerada aceitável para um planejamento pré-operatório adequado. ${ }^{23}$

Wimsey et al. ${ }^{8}$ compararam o posicionamento de uma moeda entre as coxas e a medição das espinhas ilíacas superiores anteriores no paciente com uma pinça. Os resultados do cálculo de ampliação foram favoráveis ao uso da moeda, com precisão de $98,9 \%$ e erro inferior a $0,5 \mathrm{~mm}$ nas medições digitais do marcador. Quando posicionado entre as coxas, o tamanho médio da moeda em nossa amostra foi de $25 \mathrm{~mm}$ para ambos os examinadores, o coeficiente de variação foi de no máximo 3\%, e a precisão foi entre 50 e $60 \%$. 0 erro máximo foi de $1 \mathrm{~mm}$ (4\%), provavelmente superestimado pelo fato de o software utilizado para medição não ter precisão milimétrica.

Neste estudo, os marcadores foram posicionados por um médico com experiência em identificação de proeminências ósseas, ao contrário da grande maioria dos estudos anteriores. $^{8-17,23}$ A posição mais simples foi sobre a mesa de exame, uma vez que a identificação de proeminências ósseas pode ser difícil, especialmente em pacientes obesos ou com deformidades. The et al. ${ }^{23}$ relataram a importância do posicionamento correto, demonstrando que o marcador colocado ao nível do trocânter a $1 \mathrm{~cm}$ anterior ou posterior ao quadril representa uma diferença de $1 \%$ na ampliação.

Algumas limitações deste estudo devem ser mencionadas. A perda amostral no trocânter maior provavelmente ocorreu devido à dificuldade de adaptação desse método nas almofadas de gordura laterais e posteriores em relação ao quadril, especialmente nas pacientes do sexo feminino. Alterações na anatomia superficial de pacientes submetidos a múltiplas cirurgias foi outro fator que pode ter tendenciado a precisão do posicionamento dos marcadores. Também pode ter ocorrido algum viés na precisão de calibração e cálculo do diâmetro dos marcadores, pois o software utilizado não é capaz de medir valores decimais de milímetros.

\section{Conclusão}

Recomendamos o uso da moeda colocada entre as coxas e sugerimos o uso concomitante de outra moeda, ou uma esfera, na mesa de exame, considerando a diferença de $8 \%$ menor que o tamanho real, para evitar a não visualização do marcador na imagem.

Os métodos apresentados neste estudo são úteis para calibração radiográfica no planejamento pré-operatório e podem estimar a ampliação radiográfica com margem de segurança entre $-3 \% \mathrm{e}+3 \% .{ }^{23}$ Acreditamos que a moeda pode ser usada em vez de uma esfera sem sacrificar a precisão quando aplicada clinicamente para calibração radiográfica no planejamento pré-operatório. É necessário entender as variações de tamanho dos marcadores ao redor do quadril antes de realizar a calibração radiográfica para correção correta da ampliação e para obter melhor precisão no planejamento pré-operatório. Em pacientes com obesidade mórbida, abdome em avental, deformidade do quadril ou cirurgia prévia, deve-se prestar atenção à palpação correta das estruturas ósseas para minimizar erros de posicionamento do marcador.

\section{Suporte Financeiro}

Não houve suporte financeiro de fontes públicas, comerciais, ou sem fins lucrativos.

\section{Conflito de interesses}

Os autores não têm conflito de interesses para declarar.

\section{Referências}

1 Muller ME. Total hip replacement: planning, technique and complications. In: Surgical management of degenerative arthritis of the lower limb. Philadelphia: Lea and Faber; 1975:90-113

2 Müller ME. Lessons of 30 years of total hip arthroplasty. Clin Orthop Relat Res 1992;(274):12-21

3 Knight JL, Atwater RD. Preoperative planning for total hip arthroplasty. Quantitating its utility and precision. J Arthroplasty 1992; 7(Suppl):403-409

4 Hoikka V, Paavilainen T, Lindholm TS, Turula KB, Ylikoski M. Measurement and restoration of equality in length of the lower limbs in total hip replacement. Skeletal Radiol 1987;16(06): 442-446

5 Eggli S, Pisan M, Müller ME. The value of preoperative planning for total hip arthroplasty. J Bone Joint Surg Br 1998;80(03):382-390

6 Baghdadi YM, Larson AN, Sierra RJ. Restoration of the hip center during THA performed for protrusio acetabuli is associated with better implant survival. Clin Orthop Relat Res 2013;471(10): 3251-3259

7 Iorio R, Siegel J, Specht LM, Tilzey JF, Hartman A, Healy WL. A comparison of acetate vs digital templating for preoperative planning of total hip arthroplasty: is digital templating accurate and safe? J Arthroplasty 2009;24(02):175-179

8 Wimsey S, Pickard R, Shaw G. Accurate scaling of digital radiographs of the pelvis. A prospective trial of two methods. J Bone Joint Surg Br 2006;88(11):1508-1512

9 Franken M, Grimm B, Heyligers I. A comparison of four systems for calibration when templating for total hip replacement with digital radiography. J Bone Joint Surg Br 2010;92(01):136-141

10 Archibeck MJ, Cummins T, Tripuraneni KR, et al. Inaccuracies in the Use of Magnification Markers in Digital Hip Radiographs. Clin Orthop Relat Res 2016;474(08):1812-1817

11 Conn KS, Clarke MT, Hallett JP. A simple guide to determine the magnification of radiographs and to improve the accuracy of preoperative templating. J Bone Joint Surg Br 2002;84(02):269-272

12 Petretta R, Strelzow J, Ohly NE, Misur P, Masri BA. Acetate templating on digital images is more accurate than computerbased templating for total hip arthroplasty. Clin Orthop Relat Res 2015;473(12):3752-3759

13 Gamble P, de Beer J, Petruccelli D, Winemaker M. The accuracy of digital templating in uncemented total hip arthroplasty. J Arthroplasty 2010;25(04):529-532

14 Heinert G, Hendricks J, Loeffler MD. Digital templating in hip replacement with and without radiological markers. J Bone Joint Surg Br 2009;91(04):459-462

15 González Della Valle A, Comba F, Taveras N, Salvati EA. The utility and precision of analogue and digital preoperative planning for total hip arthroplasty. Int Orthop 2008;32(03):289-294 
16 The B, Verdonschot N, van Horn JR, van Ooijen PM, Diercks RL. Digital versus analogue preoperative planning of total hip arthroplasties: a randomized clinical trial of 210 total hip arthroplasties. J Arthroplasty 2007;22(06):866-870

17 Boese CK, Lechler P, Rose L, et al. Calibration Markers for Digital Templating in Total Hip Arthroplasty. PLoS One 2015;10(07):e0128529

18 Polesello GC, Nakao TS, Queiroz MC, et al. Proposta de Padronização do Estudo Radiográfico do Quadril e da Pelve. Rev Bras Ortop 2011;46(06):634-642

19 Blake CA, van der Merwe J, Raubenheimer JE. A practical way to calibrate digital radiographs in hip arthroplasty. SA Orthop J 2013;12(04):33-37
20 Fleiss JL. The design and analysis of clinical experiments. New York: Wiley; 1986

21 Altman DG, Bland JM. Measurement in Medicine: The Analysis of Method Comparison Studies. Statistician 1983;32(03):307-317

22 Streiner DL, Norman GR. Health measurement scales: a practical guide to their development and use. 2nd ed. New York: Oxford University Press Inc; 1995

23 The B, Diercks RL, Stewart RE, van Ooijen PM, van Horn JR. Digital correction of magnification in pelvic $\mathrm{x}$ rays for preoperative planning of hip joint replacements: theoretical development and clinical results of a new protocol. Med Phys 2005;32(08): 2580-2589 